\title{
Noncommutative Harmonic Oscillator at Finite Temperature: A Path Integral Approach
}

\author{
A. Jahan \\ Department of Physics, Amirkabir University of Technology \\ P. O. Box 15875-4413, Tehran, Iran
}

Received on 2 November, 2007

\begin{abstract}
We use the path integral approach to a two-dimensional noncommutative harmonic oscillator to derive the partition function of the system at finite temperature. It is shown that the result based on the Lagrangian formulation of the problem, coincides with the Hamiltonian derivation of the partition function.
\end{abstract}

Keywords: Noncommutative space; Partition function

\section{INTRODUCTION}

Recently, there has been an immense amount of challenge devoted to the subject of noncommutative space-time. Although, the idea of noncommutating space-time coordinates is an old proposal [1], the recent discoveries in string/M theories were the main source of renewed interests in the subject [2]. In particular, much work is dedicated to study the noncommutative version of the quantum field theory (see for example [3] and references there in). In noncommutative field theories one replaces the space-time coordinates $x^{\mu}$ with operators $\hat{x}^{\mu}$, which do not commute with each other, i.e.

$$
\left[\hat{x}^{\mu}, \hat{x}^{v}\right]=i \theta^{\mu \nu}
$$

with $\theta^{\mu v}$ as a real anti-symmetric matrix. Thus because of the uncertainty relation induced by the relation (1), the short distance scales in the $x^{\mu}$ direction correspond to the large distance scales in the $x^{v}$ direction and vice versa. So there will be a mixing between the ultraviolet and infrared behaviors of the field theories in noncommutative space-times. Such a problem, which is called the UV/IR mixing problem, is characteristic of the models defined in noncommutative space [4]. In a noncommutative space-time the usual product between the fields must be replaced by the Weyl-Moyal bracket or starproduct defined as

$$
f(x) \star g(x)=\lim _{x \rightarrow y} \exp \left(\frac{i}{2} \theta^{\mu v} \partial_{\mu}^{y} \partial_{v}^{x}\right) f(x) g(x)
$$

The star-product is the usual starting point in the most studies about the noncommutative field theories since it encodes the noncommutativity of space-time for the product of the several fields defined in the same point in the following sense

$$
\hat{f}_{1}(\hat{x}) \cdots \hat{f}_{n}(\hat{x}) \rightarrow f_{1}(x) \star \cdots \star f_{n}(x)
$$

On the other hand it seems natural to explore the noncommutative version of quantum mechanics since it stands as the one-particle low energy sector of the quantum field theory [5]. The following set of relations among the coordinates and momenta (setting $\hbar=1$ )

$$
\begin{aligned}
{\left[\hat{x}^{i}, \hat{x}^{j}\right] } & =i \theta^{i j} \\
{\left[\hat{x}^{i}, \hat{p}^{j}\right] } & =i \delta^{i j} \\
{\left[\hat{p}^{i}, \hat{p}^{j}\right] } & =0
\end{aligned}
$$

characterize the noncommutative version of the quantum mechanics (Latin indices stand for the spatial coordinates). An equivalent procedure to implement the star-product is to define a new set of commutating coordinates $x^{i}$ via [5]

$$
x^{i}=\hat{x}^{i}+\frac{1}{2} \theta^{i j} p^{j}
$$

(Through the paper summation is implied over the repeated indices). Therefore for the interaction potential $\hat{U}\left(\hat{x}^{i}\right)$ defined in noncommutative space one gets the effective potential in usual commutative space as

$$
U\left(x^{i}, \bar{p}^{i}\right)=U\left(x^{i}-\theta^{i j} p^{j} / 2\right), \quad \bar{p}^{i}=\theta^{i j} p^{j}
$$

Path integral approach to the noncommutative quantum mechanics has revealed in series of the recent works [6]. It is demonstrated by the authors that how the noncommutativty of space could be implemented in Lagrangian formulation of the quantum mechanics. Here we aim to incorporate the effect of noncommutativty of space on the thermodynamics of a two dimensional harmonic oscillator by means of the formalism developed in [6]. So after a brief review of the Lagrangian aspect of noncommutative quantum mechanics we derive the partition function of the system by identifying the temperature as imaginary time. It is shown that the result is in accordance with one obtained via the Hamiltonian approach to the partition function.

\section{LAGRANGIAN FORMULATION OF NONCOMMUTATIVE QUANTUM MECHANICS}

The Hamiltonian governing the dynamics of a harmonic oscillator in noncommutative space is

$$
\hat{H}=1 / 2 m\left(\hat{p}_{1}^{2}+\hat{p}_{2}^{2}\right)+\frac{1}{2} m \omega^{2}\left(\hat{x}_{1}^{2}+\hat{x}_{2}^{2}\right)
$$

On implementing the transformation (5), one finds the effective Hamiltonian in usual commutative space as

$H=\kappa / 2 m\left(p_{1}^{2}+p_{2}^{2}\right)+\frac{1}{2} m \theta \omega^{2}\left(x_{1}^{2}+x_{2}^{2}\right)+\frac{1}{2} m \theta \omega^{2}\left(x_{1} p_{2}-x_{2} p_{1}\right)$

with $\kappa^{2}=1+m^{2} \omega^{2} \theta^{2} / 4$ and corresponding energy spectrum as

$$
\varepsilon_{n_{+}, n_{-}}=\omega\left[(\sqrt{\kappa}+\sqrt{\kappa-1}) n_{+}+(\sqrt{\kappa}-\sqrt{\kappa-1}) n_{-}+\sqrt{\kappa}\right]
$$


The corresponding Lagrangian can be achieved by means of the Legender transformation $L_{\theta}\left(x^{i}, \dot{x}^{i}\right)=p^{i} \dot{x}^{i}-H_{\theta}\left(x^{i}, p^{i}\right)$, upon replacing for the momenta from $\dot{x}^{i}=\partial H_{\theta} / \partial p^{i}$. Hence the Lagrangian associated with the Hamiltonian (8) will be

$$
L=\frac{m}{2 \kappa}\left(\dot{x}_{2}^{2}+\dot{x}_{1}^{2}\right)-\frac{m \omega^{2}}{2 \kappa}\left(x_{2}^{2}+x_{1}^{2}\right)+\frac{\theta m^{2} \omega^{2}}{2 \kappa}\left(\dot{x}_{2} x_{1}-\dot{x}_{1} x_{2}\right)
$$

The above Lagrangian admits the equations of motion as differential equations of rank four. When the solutions are inserted in (10) and integrated out over the time variable $\tau$, one finds for the action

$$
\begin{aligned}
S_{\theta}\left(\mathbf{x}^{\prime \prime}, \mathbf{x}^{\prime}, \tau\right) & =\frac{m \omega}{2 \sqrt{\kappa} \sin (\omega \tau \sqrt{\kappa})}\left[\left(\mathbf{x}^{\prime 2}+\mathbf{x}^{\prime \prime 2}\right) \cos (\omega \tau \sqrt{\kappa})\right. \\
& \left.-2\left(\mathbf{x}^{\prime} \cdot \mathbf{x}^{\prime \prime}\right) \cos (\omega \tau \sqrt{\kappa-1})+2\left(\mathbf{x}^{\prime} \times \mathbf{x}^{\prime \prime}\right)_{z} \sin (\omega \tau \sqrt{\kappa-1})\right]
\end{aligned}
$$

with boundary condition $\mathbf{x}^{\prime \prime}=\mathbf{x}(\tau)$ and $\mathbf{x}^{\prime}=\mathbf{x}(0)$. Thus one obtains the semiclassical propagator (transition amplitude) as

$$
\begin{aligned}
K_{\theta}\left(\mathbf{x}^{\prime \prime}, \tau ; \mathbf{x}^{\prime}, 0\right) & =\sqrt{\operatorname{det}\left(-\frac{\partial^{2} S_{\theta}}{\partial \mathbf{x}^{\prime \prime} \partial \mathbf{x}^{\prime}}\right)} e^{i S_{\theta}} \\
& =\frac{m \omega}{2 \pi i \sqrt{\kappa}|\sin (\omega \tau \sqrt{\kappa})|} e^{i S_{\theta}}
\end{aligned}
$$

\section{FINITE TEMPERATURE CONSIDERATIONS: PARTITION FUNCTION}

The partition function $Z(\beta)$ plays a vital role in thermodynamical considerations of the physical systems at finite temperature. It can be defined in terms of the propagator of system as [7]

$$
Z(\beta)=\operatorname{Tr} K_{\theta}\left(\mathbf{x}^{\prime \prime}, \beta ; \mathbf{x}^{\prime}, 0\right)
$$

where the inverse temperature parameter is defined as $\beta=i \tau$. The symbol $\operatorname{Tr}$ stands for the functional trace which for a bilocal function $A\left(\mathbf{x}, \mathbf{x}^{\prime}\right)$ in $\mathrm{D}$ dimensions is defined as

$$
\operatorname{Tr} A\left(\mathbf{x}, \mathbf{x}^{\prime}\right)=\int_{-\infty}^{+\infty} d^{D} x A(\mathbf{x}, \mathbf{x})
$$

When the time parameter in (12) is replaced with the inverse temperature parameter $\beta=i \tau$, the semiclasical propagator (12) modifies to

$$
K_{\theta}\left(\mathbf{x}^{\prime \prime}, \beta ; \mathbf{x}^{\prime}, 0\right)=\frac{m \omega}{2 \pi \sqrt{\kappa} \sinh (\omega \beta \sqrt{\kappa})} e^{-S_{\theta}^{E}}
$$

with

$$
\begin{aligned}
S_{\theta}^{E}\left(\mathbf{x}^{\prime \prime}, \mathbf{x}^{\prime}, \beta\right) & =\frac{m \omega}{2 \sqrt{\kappa} \sinh (\omega \beta \sqrt{\kappa})}\left[\left(\mathbf{x}^{\prime 2}+\mathbf{x}^{\prime \prime 2}\right) \cosh (\omega \beta \sqrt{\kappa})\right. \\
& \left.-2\left(\mathbf{x}^{\prime} \cdot \mathbf{x}^{\prime \prime}\right) \cosh (\omega \beta \sqrt{\kappa-1})+2\left(\mathbf{x}^{\prime} \times \mathbf{x}^{\prime \prime}\right)_{z} \sinh (\omega \beta \sqrt{\kappa-1})\right]
\end{aligned}
$$

where we have invoked the well-known trigonometric identities $\cos i \alpha=i \cosh \alpha$ and $\sinh \alpha=-i \sin i \alpha$. Thus from the definition (14) we find for the trace of the term $e^{-S_{\theta}^{E}}$

$$
\operatorname{Tr}^{-S_{\theta}^{E}}=\frac{\pi \sqrt{\kappa}}{m \omega} \frac{\sinh (\omega \beta \sqrt{\kappa})}{\cosh (\omega \beta \sqrt{\kappa})-\cosh (\omega \beta \sqrt{\kappa-1})}
$$

Therefore one is left with the partition function as

$$
\begin{aligned}
Z(\beta) & =\frac{m \omega}{2 \pi \sqrt{\kappa} \sinh (\omega \beta \sqrt{\kappa})} \operatorname{Tr}^{-S_{\theta}^{E}} \\
& =\frac{1}{2[\cosh (\omega \beta \sqrt{\kappa})-\cosh (\omega \beta \sqrt{\kappa-1})]} \\
& =\frac{1}{4 \sinh \left[\frac{\omega \beta}{2}(\sqrt{\kappa}+\sqrt{\kappa-1})\right] \sinh \left[\frac{\omega \beta}{2}(\sqrt{\kappa}-\sqrt{\kappa-1})\right]}
\end{aligned}
$$


The above result for the partition function is derived earlier in the context of Hamiltonian formulation of the problem [8]

$$
\begin{aligned}
Z(\beta) & =\operatorname{tr} e^{-\beta H_{\theta}}=\sum_{n_{+}, n_{-}=0}^{+\infty} e^{-\beta \varepsilon_{n_{+}, n_{-}}} \\
& =\frac{1}{4 \sinh \left[\frac{\omega \beta}{2}(\sqrt{\kappa}+\sqrt{\kappa-1})\right] \sinh \left[\frac{\omega \beta}{2}(\sqrt{\kappa}-\sqrt{\kappa-1})\right]}
\end{aligned}
$$

with energy spectrum given by Eq. (9). (Note that the result of above summation derived in [8] is false since the arguments of the sinh functions must be multiplied by the factor $1 / 2$ ). The free energy $F(\beta)$ of a system at finite temperature is related to the partition function as

$$
F(\beta)=-\frac{1}{\beta} \ln Z(\beta)
$$

which at low temperature limit tends to the ground state energy of the system. In particular for the case of harmonic oscillator we have

$$
\lim _{\beta \rightarrow \infty} F(\beta)=\omega \sqrt{\kappa}
$$

which coincides with the ground state energy of the system, $\varepsilon_{0,0}=\omega \sqrt{\kappa}$ (see Eq. (9)). In the limit $\theta \rightarrow 0$, the parameter $\kappa$ tends to unity and one recovers the ground state energy of usual two-dimensional harmonic oscillator.
[1] H. S. Snyder, Phys. Rev. 71, 38 (1946); C. N. Yang, Phys. Rev. 72, 874 (1947).

[2] A. Connes, M. R. Douglas, and A. Schwartz, JHEP 9802, 003 (1998); R. Szabo, Phys. Rept. 378, 207 (2003).

[3] M. Wohlgenannt, Noncommutative Geometry and Physics, arXiv: hep-th/0602105; M. Wohlgenannt, Introduction to a noncommutative version of the standard model, arXiv: hepth/0302070; H. O. Girotti, Noncommutative Quantum Field Theory, arXiv: hep-th/0301237; J. M. Carmona, J. L. Cortes, J. Gamboa, and F. Mendez, JHEP 0303, 058 (2003).

[4] T. Filk, Phys. Lett. B 376, 53 (1996).

[5] A. E. F. Djemai, H. Smail, Commun. Theor. Phys. 41, 837 (2004); J. Gamboa, M. Loewe, F. Mendez, and J. C. Rojas, Mod. Phys. Lett A 16, 2075 (2001); M. Demetrian, D. Kochan, Acta.
Physica. Slovaca 52, 1 (2002); J. Gamboa, M. Loewe, F. Mendez, and J. C. Rojas, Phys. Rev. D 64, 067901 (2001); L. Mezincescu, Star Operation in Quantum Mechanics, arXiv: hep-th/0007046.

[6] C. Acatrinei, Path Integral Formulation of Noncommutative Quantum Mechanics, JHEP 0109, 007 (2001); B. Dragovic, Z. Rakic, Theor. Math. Phys. 140, 1299 (2004); B. Dragovic, Z. Rakic, Path Integral Approach to Noncommutative Quantum Mechanics, arXiv: hep-th/0401198.

[7] C. Grosche, F. Steiner, Handbook of Feynman Path Integrals, Springer-Verlag, Berlin, 1998.

[8] J. Gamboa, M. Loewe, F. Mendez, and J. C. Rojas, Int. J. Mod. Phys. A 17, 2555 (2002). 